\title{
Spectroscopic and biochemical characteristics of flax transgenic callus cultures producing PHB
}

\author{
Magdalena Wróbel-Kwiatkowska ${ }^{1} \cdot K$ Kamil Kostyn ${ }^{2} \cdot$ Lucyna Dymińska $^{3} \cdot$ Jerzy Hanuza $^{4} \cdot$ Adam Kurzawa $^{5}$. \\ Magdalena Żuk ${ }^{2}$. Waldemar Rymowicz ${ }^{1}$
}

Received: 22 November 2019 / Accepted: 2 March 2020 / Published online: 10 March 2020

(c) The Author(s) 2020

\begin{abstract}
Linum usitatissimum $L$. is a plant used by human since ancient times. Presently flax has both industrial and nutritional significance. The programmes of restoration of flax cultivation and processing are implemented to renew the importance of this plant for agriculture and economy. Genetic engineering methods and techniques of plant tissue cultures can answer this requirement, because they give the opportunity for obtaining of new plants and novel application of them. In the present study the impact of genetic modification of flax plants on the ability of morphogenesis was investigated. The subject of this research remain genetically modified flax plants enriched in poly- $\beta$-hydroxybutyrate (PHB), interesting biodegradable compound showing bioactive properties and acting as anti-inflammatory and anti-microbial factor. Another aim of this study was to characterize chemical and structural properties of callus derived from the engineered plants in comparison to the data obtained for non-transgenic, control callus cultures. The presence of PHB was confirmed by FTIR technique and it was suggested that this polymer may have impact on about $20 \%$ higher biomass growth observed for transgenic callus cultures in comparison to control cultures. The level of phenolic compounds was not significantly changed in the transgenic calluses when compared to wild-type cultures. The extracellular matrix (EMC) was noticed for the surface of genetically modified calluses, such structure was not visible for calluses derived from the control cultures. The cellulose in plant cell walls of transgenic calluses was structurally changed, it was not as organized as in control callus, resulting in lower crystallinity index values.
\end{abstract}

\section{Key message}

1. FTIR and SEM analyses revealed differences between callus producing PHB when compared to wild-type callus.
2. PHB might influence the proliferative properties of plant cells.

Keywords Flax $\cdot$ Callus $\cdot$ Polyhydroxybutyrate (PHB) · Fourier-transform infrared spectroscopy (FTIR) $\cdot$ Scanning electron microscopy (SEM)

Communicated by Sergio J. Ochatt.

Magdalena Wróbel-Kwiatkowska

magdalena.wrobel-kwiatkowska@upwr.edu.pl

1 Department of Biotechnology and Food Microbiology, Wrocław University of Environmental and Life Sciences, Chełmońskiego Str. 37, 51-630 Wrocław, Poland

2 Faculty of Biotechnology, University of Wrocław, Przybyszewskiego 63/77, 51-148 Wroclaw, Poland
3 Department of Bioorganic Chemistry, Wroclaw University of Economics and Business, Komandorska 118/120, 53-345 Wrocław, Poland

4 Institute of Low Temperature and Structure Research, Polish Academy of Sciences, P.O. Box 1410, 50-950 Wrocław, Poland

5 Department of Lightweight Elements Engineering, Foundry and Automation, Wroclaw University of Science and Technology, Smoluchowskiego 25, 50-370 Wrocław, Poland 


\section{Introduction}

Flax (Linum usitatissimum L.) is a plant with very long breeding traditions dating back to ancient times. New technologies concerning transgenesis give the opportunity to modify flax plants and their products, especially that this plant species can be easily transformed by Agrobacterium tumefaciens (Ludvíková and Griga 2015). The first study regarding the transformation of flax by using of A. tumefaciens was published in 1983 (Hepburn et al. 1983). It should be pointed out that flax is characterized with very low genetic variability, because it is self-pollinated, thus transgenesis might be the method for improving its properties and increasing genetic diversity.

Nowadays two main directions of flax research can be distinguished: one concerning flax transformation resulting in the improvement of qualitative or quantitative properties for example increasing of polyunsaturated fatty acids in flax seeds (Du et al. 2019) and second related to in vitro cultures of flax cells and induction of secondary metabolites synthesis. The example for this type of research may be the application of chemogenic silver nanoparticles in flax cultures for obtaining increased level of lignans and neolignans (Zahir et al. 2019).

In the present study another strategy was applied. The transgenic flax plants were used for the induction and characterization of callus suspension cultures. The object of this research was transgenic flax (L. usitatissimum L.) with overexpression of three genes (EMBL/database Acc. Nos. J04987, J04987, J05003) derived from Cupriavidus necator (formerly called Ralstonia eutropha) and coding three enzymes respectively: $\beta$-ketothiolase, acetoacetyl-CoA reductase, PHB synthase, crucial for poly-beta-hydroxybutyrate (PHB) synthesis. PHB is considered as green polymer, because its chemical and physical properties are similar to polypropylene, but in contrast to it PHB is biodegradable, because it is synthesized by many genera of bacteria (Poirier et al. 1995; Raza et al. 2018). The synthesis of polymers in plants is an interesting issue and it can be mainly performed not for the production of these compounds, but rather to change plant properties. Recently it has been shown that PHB oligomers may have antifungal and antibacterial properties (Ma et al. 2019), and that amino-PHB polymers may acts as anti-cancer and antimicrobial agents (Abdelwahab et al. 2019).

Two different methods for the production of polymers were applied in flax (Wróbel et al. 2004; Wróbel-Kwiatkowska et al. 2019). Initially, the simplest form of polyhydroxyalkanoates (PHA) i.e. PHB was synthesized in flax plants after introduction of three genes from PHB biosynthetic pathway, while the latest research concerned mcl-PHA (medium-chain-length PHA) synthesis in this plant. The modified plants used in the present study exhibited PHB synthesis and showed unchanged phenotype, improved mechanical properties of stems and fibres (especially observed for one modified line M13) (Wróbel-Kwiatkowska et al. 2009a). The synthesis of PHB resulted in higher content of cellulose, decrease in lignin and pectin amount as well as hemicellulose constituents (Wróbel-Kwiatkowska et al. 2007a, b). Transgenic plants accumulated higher level of phenolic compounds than untransformed control plants and exhibited higher resistance to pathogen infection. Cellulose in the transgenic plants was structurally changed and formed hydrogen and ester bonds with PHB (Wróbel-Kwiatkowska et al. 2009b). One of the transgenic lines with the highest PHB level (M50) was used as the source of fibres for composite preparation (Wróbel-Kwiatkowska et al. 2012) and the basis for micronization (Dymińska et al. 2012). Fibres isolated from the modified $\mathrm{M}$ plants were also applied as promising biomaterial for wound healing (Gąsiorowski et al. 2019).

In the present research stable transformants from in vitro cultures, overexpressing genes from PHB synthesis route were applied to establish and characterize callus suspension cultures. The aim of this study was to investigate the impact of the transgenesis on the chemical and structural properties of callus cultures obtained from genetically engineered plants. The quantitative analysis of phenolic compounds was performed in the M50-derived callus cultures and compared to wild-type cultures. The presence of PHB in the transgenic callus was confirmed by infrared spectroscopy. The rate growth of the callus biomass showed higher values for the transgenic cultures than for control. Scanning electron microscopy (SEM) analysis revealed the presence of extracellular matrix (EMC) on the surface of transgenic callus cells, which was not observed for the wild-type callus. Spectroscopic data obtained via Fourier-transform infrared spectroscopy (FTIR) indicated the decrease in the crystallinity index of cellulose in callus derived from the transgenic (M50) cultures when compared to control callus cultures.

\section{Materials and methods}

\section{Plant material and growth conditions}

The object of the present research was flax (Linum usitatissimum L.) of fibre cultivar Nike. The seeds of wild type plants were provided by the Flax and Hemp Collection of the Institute of Natural Fibres, in Poland. Transgenic M50 flax plants were generated previously (Wróbel et al. 2004) and grown in the tissue cultures. The genetically modified flax plants (M50) were created by transformation performed via Agrobacterium tumefaciens method and resulted in the introduction of three genes encoding: $\beta$-ketothiolase, 
acetoacetyl-CoA reductase and PHB synthase. All of the genes derived from Cupriavidus necator (syn. Ralstonia eutropha) and they were necessary for PHB synthesis.

The plants were grown under in vitro culture conditions in the phytotron (at photoperiod $16 \mathrm{~h} / 8 \mathrm{~h}$, at $21{ }^{\circ} \mathrm{C} / 16{ }^{\circ} \mathrm{C}$ ) on a MS medium (Murashige and Skoog 1962), supplemented with $1 \%$ sucrose and solidified with $0.8 \%$ agar (Sigma) (pH 5.8). Plant Preservative Mixture (PPM ${ }^{\mathrm{TM}}$, Plant Cell Technology) was added to the medium at a concentration of $375 \mu \mathrm{l} \times 500 \mathrm{ml}^{-1}$.

\section{Callus initiation from flax plants}

Callus was generated from selected 4-week-old flax plants (genetically modified and control, wt) propagated via in vitro cultures. The leaves and stem fragments of the plants were transferred to callus induction medium containing MS medium supplemented with $2.5 \%$ sucrose, $2.5 \%$ glucose, $4.44 \mu \mathrm{M}$ BAP, $0.26 \mu \mathrm{M}$ NAA and solidified with $0.8 \%$ agar (Sigma) (pH 5.8). Explants were transferred to the fresh medium every 7 days until they formed callus tissue (Wróbel-Kwiatkowska et al. 2007a, b; Rutkowska-Krause et al. 2003). Initiated callus was applied to perform the suspension cultures. After achievement of the growth log phase, several pieces of wt and M50 calluses were transferred on shoot induction medium (Wróbel-Kwiatkowska et al. 2007a, b).

\section{Callus suspension cultures}

The cultures were initiated from callus derived from transgenic (M50) and wild-type (wt) flax plants (cv. Nike). Obtained calluses were cultured in a liquid MS medium supplemented with sucrose (3\%) and phytohormones $(8.88 \mu \mathrm{M}$ BAP, $2.68 \mu \mathrm{M}$ NAA) (Hano et al. 2006). The suspension cultures were maintained for 18 days in the darkness at $25^{\circ} \mathrm{C}$ on the rotary shaker (at speed of $100 \mathrm{rpm}$ ). After ten days the callus was transferred to fresh medium (the composition was the same as described above). From the 18 days-old cultures tissue was collected, weighed and analyzed.

\section{Determination of plant biomass}

Callus tissue was harvested at the same time from each culture and weighed in the sterile conditions. The relative growth rate of biomass was calculated as the difference between final and initial biomass amount.

\section{Quantitative analysis of phenolic compounds}

Lyophilized tissue of callus, cultured for 18 days, was extracted three times with methanol for $15 \mathrm{~min}$. in ultrasonic bath. The obtained pellet was hydrolyzed with $2 \mathrm{M} \mathrm{NaOH}$, then $\mathrm{pH}$ was adjusted to 3.0 and extracted three times with ethyl acetate. Both the collected methanol extracts and combined ethyl acetate extracts were dried under nitrogen flow, resuspended in methanol and analyzed using UPLC (Waters Acquity UPLC) system with a 2996 PDA QTOF mass detector on an Acquity UPLC BEH C18 $(2.1 \times 100 \mathrm{~mm}, 1.7 \mu \mathrm{m})$ column. The mobile phase was $0.1 \%$ formic acid and $100 \%$ acetonitrile (Hasiewicz-Derkacz et al. 2015).

\section{FTIR analysis}

All FTIR spectra were measured in the spectral range between $350 \mathrm{~cm}^{-1}$ and $4000 \mathrm{~cm}^{-1}$ using a Thermo Scientific Nicolet 6700 FT-IR spectrophotometer with a $2 \mathrm{~cm}^{-1}$ resolution (Wróbel-Kwiatkowska et al. 2019). Each recorded FTIR spectrum was measured using 128 scans. The spectroscopic measurements were independently repeated three times for each sample. Poly-3-hydroxybutyric acid was commercially obtained (Sigma-Aldrich) and used as a standard.

The lyophilized callus samples were prepared in the $\mathrm{KBr}$ pellet. All spectra were analyzed using computer program ORIGIN 7.5. Lorentzian distribution function was used for the data fitting. The fitting parameter $\chi^{2}$ was of order $10^{-6}$. Due to short time of measurements the moisture of the studied samples remained unchanged during the spectra acquisition, thus the residual water was not present in the studied samples.

\section{SEM (scanning electron microscopy) analysis}

Several pieces of callus collected after 18 day of culture were analyzed via scanning electron microscopy. The samples were frozen in liquid nitrogen and then freeze-dried, but chemical fixation method was not applied to avoid occurrence of artifacts (Konieczny et al. 2005). The specimens were covered with a carbon coating using Q150T sputter with turbomolecular pump from Quorum Technologies company. All the observations were done by scanning electron microscope from Hitachi TM-3000 company with EDS system.

\section{Results and discussion}

\section{The growth of callus}

Two types of calluses derived from modified flax plants (M50) and wild-type plants were achieved as described in "Materials and methods" section. The compact and green callus was derived and used for preparation of suspension cultures. There were no changes in the morphology of callus when wild type samples were compared to the transgenic explants. The biomass and growth rate of the generated callus cultures were determined and a higher rate of biomass 
increase for transgenic callus than for unmodified, control in the case of 10 days and 18 days of culturing was noticed (Fig. 1a). Finally 2.3-fold increase of the average biomass for transgene derived cultures was noticed and this parameter was higher than for wild-type callus, for which 1.8-fold growth of biomass was measured (Fig. 1b). The growth curve for the obtained cultures exhibited typical sigmoidal shape, the intensive cells divisions were noticed to 10 day of growth, which resulted in the biomass increase and after 10 day of growth the most of cultures achieved stationary phase (except one line: M50-3 \#4), in this phase the stable biomass amount was noticed. The reason for the observed difference in culture growth rate might be the genotype of flax plants used in present study, previous studies showed that there was a dependence between the genotype of plants and various culture response to the same conditions (Fiuk and Rybczyński 2008). In the present study the transgenic and non-transformed plants were used to generate callus and suspension cultures with the same methods and under the same conditions, therefore noticed changes may be in fact the result of modifications in the genome.

Thus, after 18 days of the experiment the calluses were harvested and analyzed, some of them derived either from

a

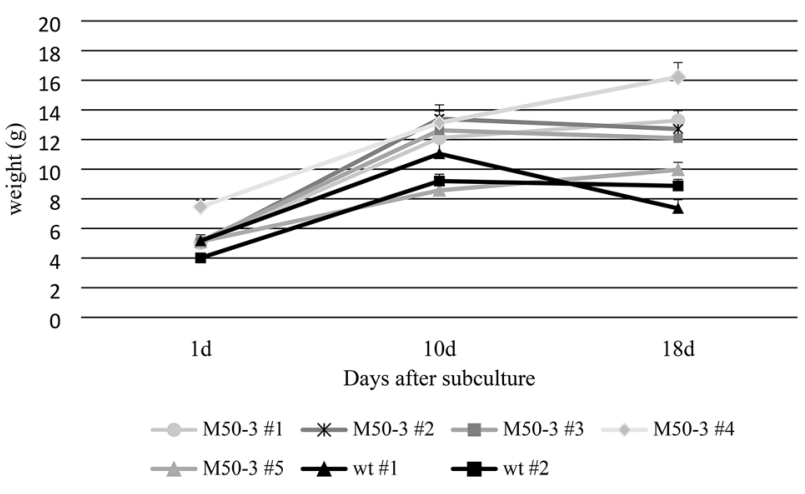

b

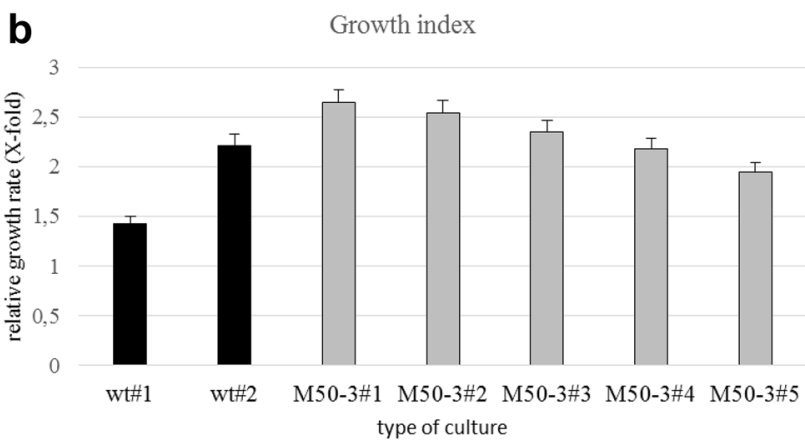

Fig. 1 a Biomass determination in callus suspension cultures, callus derived from transgenic flax plants (M50), producing PHB were compared to callus cultures, generated from unmodified flax plants (wt). b The growth rate estimated by harvested fresh weight plant biomass and calculated per inoculated biomass
M50 or wild-type flax plants, were transferred on shoot regeneration medium, but unfortunately no regenerants were obtained. Thus, the explants from generated transgenic and wild-type callus suspension cultures lost morphogenetic ability.

\section{Determination of phenolic compounds in callus suspension cultures}

Plant suspension cultures are characterized by fast growth of undifferentiated cells, which make them valuable systems for the production of many important secondary metabolites (Kolewe et al. 2008; Ali et al. 2013).

In our previous study it was shown that PHB synthesis in flax plants caused about $30 \%$ increase in the level of phenolic compounds (Wróbel-Kwiatkowska et al. 2007a, b), thus in the present paper these metabolites were determined in transgenic and control flax callus cultures via UPLC method (Fig. 2). Analyzed calluses did not show statistically important changes in the quantity of measured phenolic compounds. The highest level of these metabolites was measured for vanillin and it was slightly higher for transgenic callus than for the wild-type. In the previous research, $15 \%$ increase in the $p$-coumaric acid level was noticed for whole transgenic plants producing PHB, while ferulic acid level was not changed when compared to control

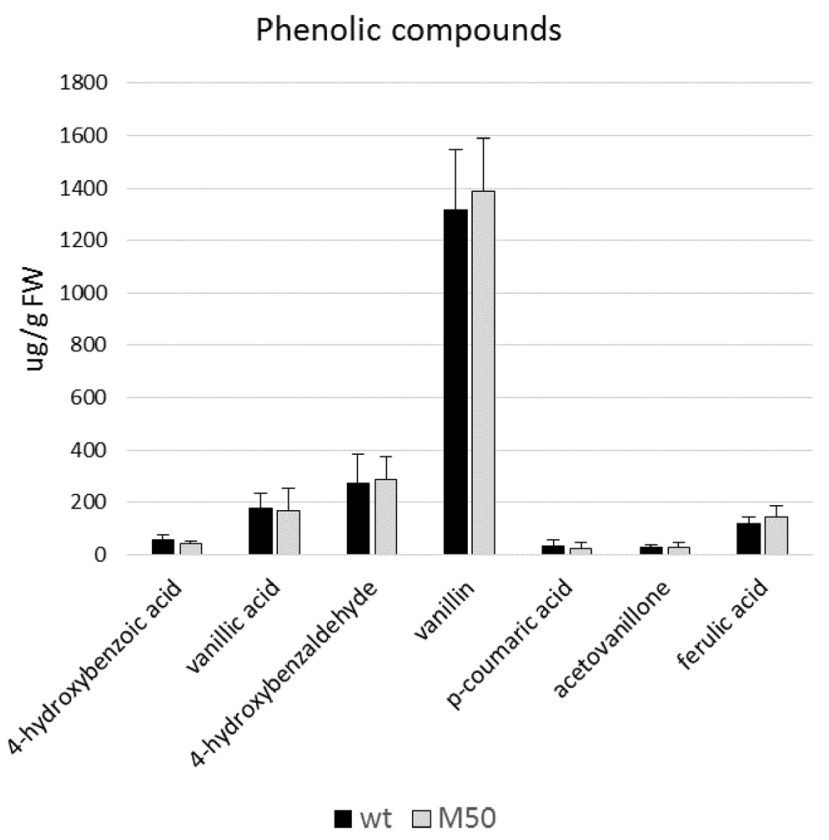

Fig. 2 The composition of phenolic compounds measured in the investigated calluses, derived from engineered flax plants (M50) and control flax (wt). The explants were obtained from suspension cultures. The data $( \pm \mathrm{SD})$ resulted from three samples per line. The statistical analysis was performed by Student's t-test $(* \mathrm{P}<0.05$; $* * \mathrm{P}<0.01)$ 
plants. A different pattern i.e. slight increase in ferulic acid amount and reduction in $p$-coumaric acid was observed in the transgenic callus (M50) in comparison to the wild-type. The level of the other investigated compounds was lower or unchanged in the M50-derived callus when compared to control explants. All the changes were however not statistically significant. Thus, transgenic flax callus cultures did not exhibit the same tendency as maternal plants enriched in PHB. Interestingly, the metabolites from vanillin synthesis pathway (ferulic acid, 4-hydroxybenzylaldehyde) were slightly increased in comparison to wild-type samples.

\section{Spectroscopic analysis of callus suspension cultures}

FTIR analysis of the callus from the control (wt) and transgenic (M50) flax showed five characteristic ranges at: 2500-4000, 1480-1800, 1190-1480, 900-1190 and 400-900 $\mathrm{cm}^{-1}$ (Fig. 3a). The contour in the range $2800-3000 \mathrm{~cm}^{-1}$ has been deconvoluted and the intensity of the component at $2940 \mathrm{~cm}^{-1}$ was used as a reference point. This wavenumber corresponds to the $\nu\left(\mathrm{CH}_{3}, \mathrm{CH}_{2}\right)$ vibration.

In the IR spectrum the broad absorption band at $3400 \mathrm{~cm}^{-1}$ is defined as band connected with hydroxyl groups engaged in forming hydrogen bonds (intramolecular and intermolecular). The absorbance in the range of $3000-3800 \mathrm{~cm}^{-1}$ revealed differences between control and transgenic samples. Thus, four Lorenzian component peaks (Table 1) were observed for control callus samples, while in the spectrum of M50 samples two additional components were noticed at $3320 \mathrm{~cm}^{-1}$ and $3052 \mathrm{~cm}^{-1}$ (Table 1). It suggests forming of new hydrogen bonds in M50 samples connected with the presence of poly-3-hydroxybutyric acid.

The range of $1600-1800 \mathrm{~cm}^{-1}$ of IR spectra is characteristic for $\mathrm{COO}^{-}$vibrations and in this range three Lorentzian components were measured in both control and M50 samples (Fig. 3b). The component at about $1740 \mathrm{~cm}^{-1}$ corresponds to the $\nu_{\text {as }}(\mathrm{COO})$ vibrations of the unconjugated carboxyl group while the band at about $1656 \mathrm{~cm}^{-1}$ corresponds to the $\nu_{\text {as }}(\mathrm{COO})$ vibration of the conjugated carboxyl group. The third band, which appears at about $1620 \mathrm{~cm}^{-1}$, corresponds to the $\nu_{\mathrm{s}}(\mathrm{COO})$ vibrations of the carboxyl group. The integral intensities analyzed for these bands for the wild-type and M50 samples showed the following trend for all components $\mathrm{I}_{\mathrm{M} 50}>\mathrm{I}_{\mathrm{WT}}$ (Fig. 3b). For the M50 sample the multiplet in the $1600-1800 \mathrm{~cm}^{-1}$ range corresponds to the $v(\mathrm{COO})$ vibrations of the carboxyl groups presented in the $\mathrm{PHB}$ and in other acids, mainly pectin components. So dependence $\mathrm{I}_{\mathrm{M} 50}>\mathrm{I}_{\mathrm{WT}}$ means higher amount of PHB and pectin.

The integral intensities of the $\delta(\mathrm{OH} \cdots \mathrm{O})$ band at about $1148 \mathrm{~cm}^{-1}$ were higher for transgenic samples than measured for control callus (Fig. 3c). These data also confirmed that the M50 sample contained more hydrogen interactions originating with the appearance of PHB.

The band at $1060 \mathrm{~cm}^{-1}$ is derived from the $\nu(\mathrm{COC})$ vibrations and the integral intensities analyzed for this band was also higher for M50 samples when compared to control, wild-type callus (Fig. 3c). It suggests that in the M50 callus additional COC bridges appear.

The component occurring at about $1270 \mathrm{~cm}^{-1}$ corresponds to $v($ COC) vibration of PHB (Lupoi et al. 2015) and the integral intensity of this band for transgenic sample was higher than for control suggesting the presence of polymer (Fig. 3d).

The IR bands in the $1300-1200 \mathrm{~cm}^{-1}$ range may be used for identification of the changes of lignin content in plant samples (Dai and Fan 2011). It can be deconvoluted into six Lorentzian components at about 1286, 1270, 1260, 1243, $1231 \mathrm{~cm}^{-1}$ and $1220 \mathrm{~cm}^{-1}$ (Fig. 3d).

The components observed at the wavenumbers $1260 \mathrm{~cm}^{-1}$ and $1243 \mathrm{~cm}^{-1}$ were close for the control and transgenic (M50) callus, indicating that the level of this polymer is comparable.

The IR bands observed at 1550 and $1515 \mathrm{~cm}^{-1}$ were described as pectin and lignin-related absorbance (WróbelKwiatkowska et al. 2009b). Their integral intensities are higher for M50 than for control sample. Now because it is known that M50 and wild-type samples contain similar amount of lignin, it means that the relative amount of pectin is larger in transgenic sample.

An important factor that characterizes the structural ordering of cellulose is the crystallinity index, Icr (KruerZerhusen et al. 2018). Icr was calculated as the intensity ratio of the $1370 \mathrm{~cm}^{-1}$ and $2900 \mathrm{~cm}^{-1}$ IR bands and it reached the values 0.48 for wild-type and 0.29 for the M50 callus. These results differed in comparison to the data determined for fibres, since transgenic fibres of M50 type exhibited increase in Icr index (achieving values 0.71-0.75) in comparison to control fibres (0.62) (Wróbel-Kwiatkowska et al. 2009a, b), such tendency was not noticed for transgenic callus. Thus, FTIR analysis confirmed the presence of PHB in the transgenic callus and showed changes in the structural properties of cellulose.

\section{The morphology of callus}

The morphology of M50 callus was similar to callus obtained from wild-type plants, i.e. in both cases callus contained large, highly vacuolated cells with irregular shape (data not shown). The plant cell wall participates in the response of plants to the environmental conditions and it is also crucial for the formation of new organs. Thus, the surface of calluses 
Fig. 3 a IR spectra of wild-type and M50 callus derived from suspension cultures. b Changes of the integral intensities of the specified bands in the range $1600-1800 \mathrm{~cm}^{-1}$. c The integral intensities of bands at $1270 \mathrm{~cm}^{-1}, 1148 \mathrm{~cm}^{-1}$, $1060 \mathrm{~cm}^{-1}$ assigned to control callus (wt) and M50 callus. d The IR bands in the range $1300-1200 \mathrm{~cm}^{-1}$ deconvoluted into six Lorentzian components a

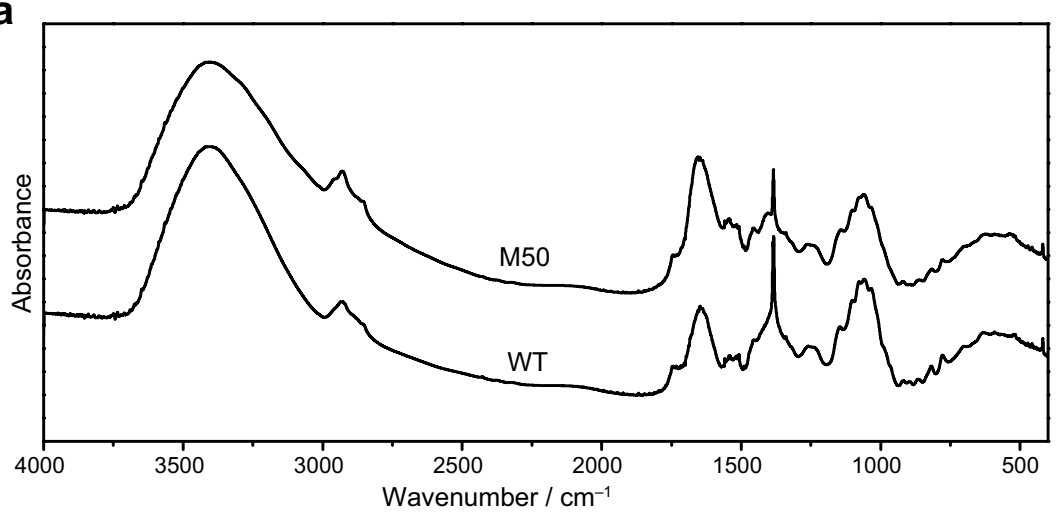

b

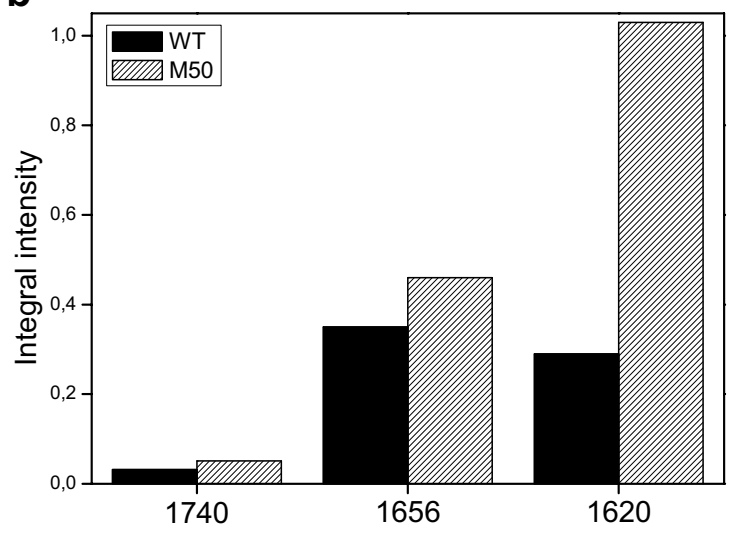

C

Wavenumber $/ \mathrm{cm}^{-1}$

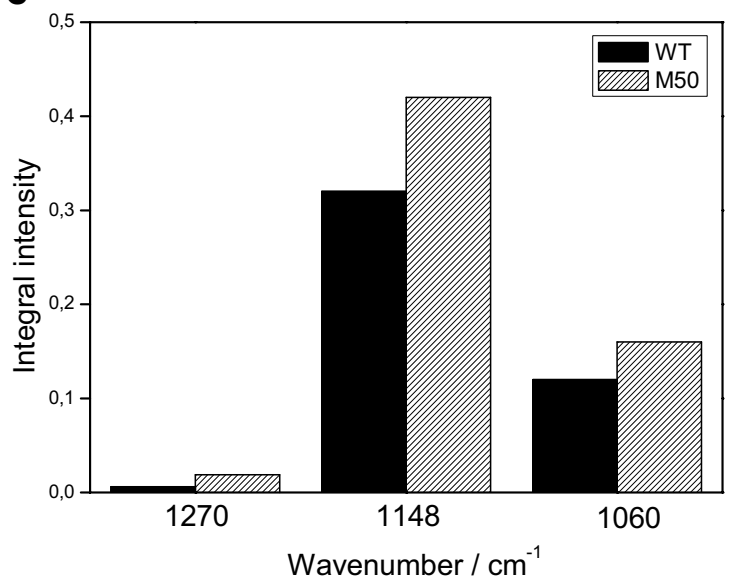

d
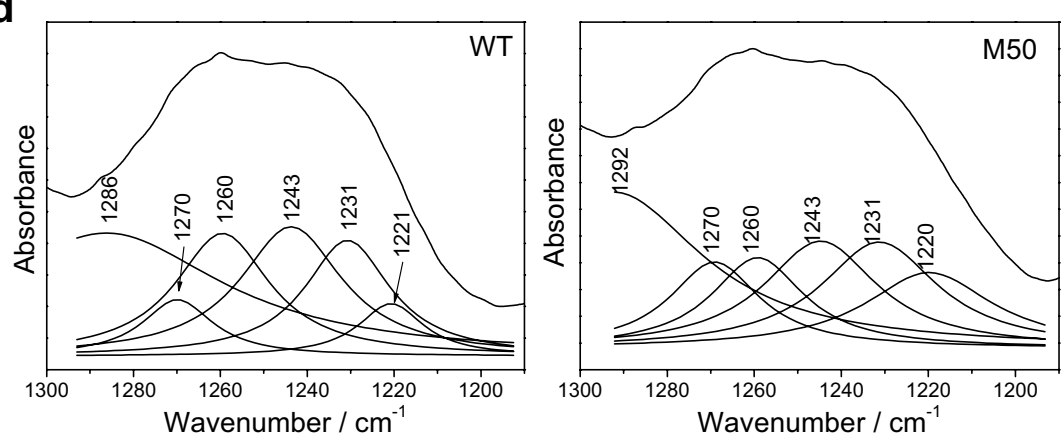
Table 1 Wavenumbers $\left(\mathrm{cm}^{-1}\right)$ of the Lorentzian components derived for the multiplet at $3000-3800 \mathrm{~cm}^{-1}$

\begin{tabular}{lll}
\hline No. & Wt & M50 \\
\hline 1 & 3565 & 3565 \\
2 & 3482 & 3479 \\
3 & 3397 & 3400 \\
4 & - & 3320 \\
5 & 3260 & 3220 \\
6 & - & 3052 \\
\hline
\end{tabular}

was investigated via SEM method (Fig. 4). The changes in the cell wall structure were noticed when transgenic callus was examined in comparison to wild-type samples. In the case of M50-derived callus extracellular matrix (EMC) was observed (Fig. 4a), this structure formed a cover of cell surface and could be connected with receiving of the morphogenic competence (Popielarska-Konieczna et al. 2008). In contrast to this observation electronograms of callus derived from wild-type samples did not show these structures (Fig. 4b). EMC matrix is a structure that has a significance in the perception of signals and may constitute plant response to the differing conditions (Popielarska-Konieczna et al. 2010). In both tested types of calluses no fibrillar structures on the surface of tissue were detected, which may indicate the lack of regeneration ability of analyzed callus. The presence of such structures is a known sign for high ability of morphogenesis (Bevitori et al. 2014; Kim 2015). Thus, formation of extracellular matrix surface network (ECMSNT) could be crucial for morphogenesis (Konieczny et al. 2005; Popielarska-Koniecznaet al. 2008) and this arrangement was not seen in the investigated samples.

The reason for the loss of regenerative capability might be the genotype of tested explants, composition and parameters of the culture, stadium of plant development, source and age of plant explant or even the size of explant etc. (Yildiz et al. 2002, 2011; Yildiz 2012).

\section{a $\mathrm{M} 50$}

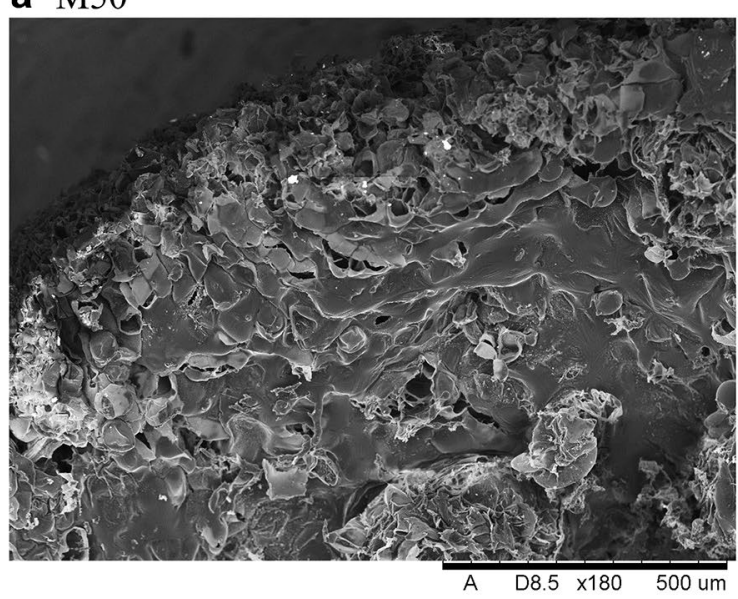

b wt

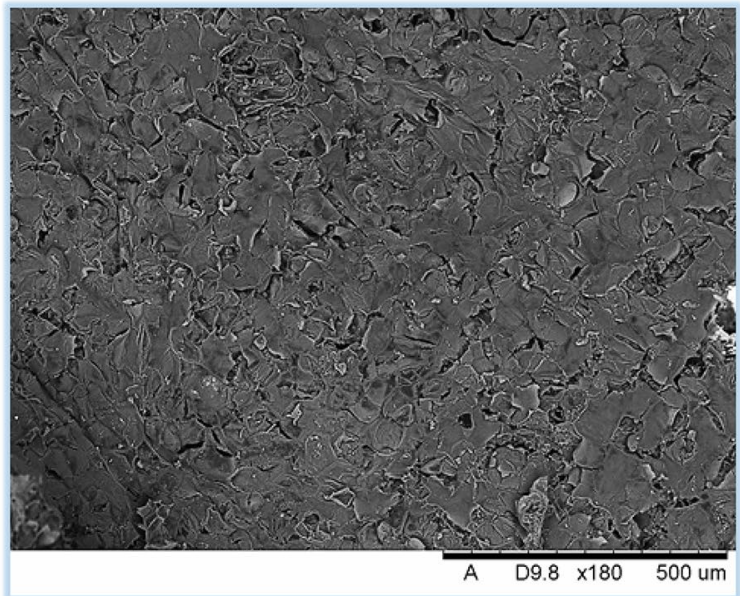

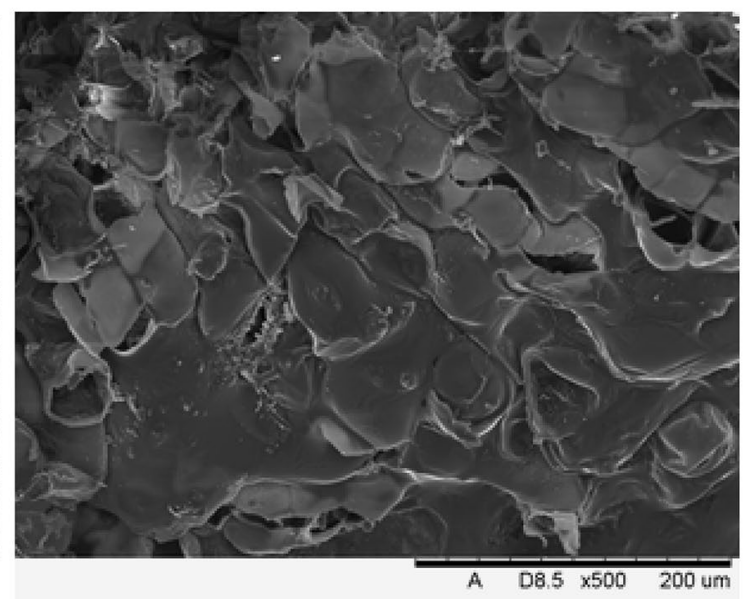

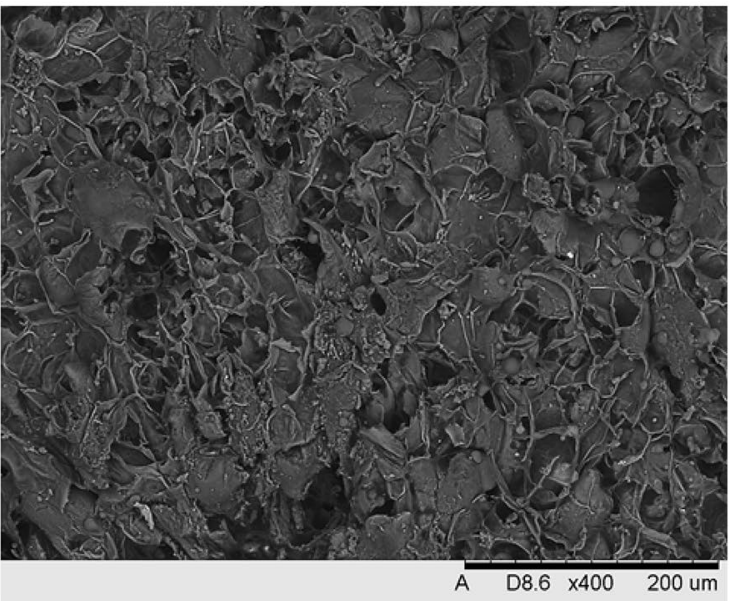

Fig. 4 The surface of callus generated from M50 (a) and wild-type (b) flax plants and derived from suspension cultures. The observations were performed with a scanning electron microscope. The electron micrographs were taken as described in "Materials and methods" section 


\section{Conclusions}

Herein, we reported the callogenesis induction on transgenic flax plants producing polymer PHB. Although the genetically engineered plants were able to generate callus, the morphogenetic ability was completely reduced and calluses were not able to regenerate into new plants. Obtained transgenic and wild-type callus cultures differed from maternal plants in the aspect of chemical composition (phenolic compound content) and structural properties measured by FTIR method (i.e. Icr). The observation made by SEM analysis showed that calluses of both types did not form the extracellular matrix surface network (ECMSN). However, for explants from the transgenic callus the formation of extracellular matrix (ECM) on the callus surface was noticed. These cultures were also characterized by about $20 \%$ higher increase of biomass than for cultures of unmodified, control calluses. Thus, synthesis of PHB may have a positive effect on the proliferation of plant cells. The similar effect of fibres containing PHB on human cells (keratinocytes and fibroblasts) was noticed and described (Gąsiorowski et al. 2019). Thus, further experiments that focus on elicitor supplementation to callus suspension cultures will be done in the nearest future and the impact of PHB on plant cell proliferation will be assessed.

Acknowledgements The authors wish to thank Dr. Michał Piegza for the freeze-drying of callus samples and Professor Jan Szopa for valuable comments. We would like to thank Professor Marzena PopielarskaKonieczna for help with interpretation of SEM results.

Author contributions The idea of this study, performing of tissue cultures experiments, data analyzing and writing of the manuscriptMWK; performing of UPLC analysis and participation in ms corrections-KK; performing of the FTIR analysis-LD; interpretation of spectroscopic data- LD, JH; performing of SEM-AK; participations in the discussion-MŻ, WR.

\section{Compliance with ethical standards}

Conflict of interest The authors declare that they have no conflict of interest.

Open Access This article is licensed under a Creative Commons Attribution 4.0 International License, which permits use, sharing, adaptation, distribution and reproduction in any medium or format, as long as you give appropriate credit to the original author(s) and the source, provide a link to the Creative Commons licence, and indicate if changes were made. The images or other third party material in this article are included in the article's Creative Commons licence, unless indicated otherwise in a credit line to the material. If material is not included in the article's Creative Commons licence and your intended use is not permitted by statutory regulation or exceeds the permitted use, you will need to obtain permission directly from the copyright holder. To view a copy of this licence, visit http://creativecommons.org/licenses/by/4.0/.

\section{References}

Abdelwahab MA, El-Barbary AA, El-Said KS, El Naggar SA, ElKholy HM (2019) Evaluation of antibacterial and anticancer properties of poly(3-hydroxybutyrate) functionalized with different amino compounds. Inter J Biol Macromol 122:793-805

Ali M, Abbasi BH, Haq IU (2013) Production of commercially important secondary metabolites and antioxidant activity in cell suspension cultures of Artemisia absinthium L. Ind Crop Prod 49:400-406

Bevitori R, Popielarska-Konieczna M, dos Santos EM, Grossi-de-Sá MF, Petrofeza S (2014) Morpho-anatomical characterization of mature embryo-derived callus of rice (Oryza sativa L.) suitable for transformation. Protoplasma 251:545-554

Dai D, Fan M (2011) Investigation of the dislocation of natural fibers by Fourier-transform infrared spectroscopy. Vib Spectrosc 55:300-306

Du C, Chen Y, Wang K, Yang Z, Zhao C, Jia Q, Taylor DC, Zhang M (2019) Strong co-suppression impedes an increase in polyunsaturated fatty acids in seeds overexpressing FAD2. J Exp Bot 70:985-994

Dymińska L, Szatkowski M, Wróbel-Kwiatkowska M, Zuk M, Kurzawa A, Syska W, Gągor A, Zawadzki M, Ptak M, Mączka M, Hanuza J, Szopa J (2012) Improved properties of micronized genetically modified flax fibers. J Biotechnol 164:292-299

Fiuk A, Rybczyński JJ (2008) Genotype and plant growth regulatordependent response of somatic embryogenesis from Gentiana spp. leaf explants. In Vitro Cell Dev Biol Plant 44:90-99

Gąsiorowski K, Gębarowski T, Moreira H, Kulma A, Szatkowski M, Szopa J (2019) Impact of fabrics from transgenic flax on cultures of skin cells. Adv Clin Exp Med 28(4):431-438

Hano C, Addi M, Bensaddek L et al (2006) Differential accumulation of monolignol-derived compounds in elicited flax (Linum usitatissimum) cell suspension cultures. Planta 223:975-989

Hasiewicz-Derkacz K, Kulma A, Czuj T, Prescha A, Żuk M, Grajzer M, Łukaszewicz M, Szopa J (2015) Natural phenolics greatly increase flax (Linum usitatissimum) oil stability. BMC Biotechnol 15:62

Hepburn AG, Clarke LE, Blundy KS, White J (1983) Nopaline Ti-plasmid, pTiT37, T-DNA insertions into a flax genome. J Mol Appl Genet 2:211-224

Kim YW (2015) Initiation of embryogenic callus from mature zygotic embryos in Japanese larch (Larix kaempferi). J Plant Biotechnol 42:223-227

Kolewe ME, Gaurav V, Roberts SC (2008) Pharmaceutically active natural product synthesis and supply via plant cell culture technology. Mol Pharm 5:243-256

Konieczny R, Bohdanowicz J, Czaplicki AZ, Przywara L (2005) Extracellular matrix surface network during plant regeneration in wheat anther culture. Plant Cell Tissue Organ Cult 83(2):201-208

Kruer-Zerhusen N, Cantero-Tubilla B, Wilson DB (2018) Characterization of cellulose crystallinity after enzymatic treatment using Fourier transform infrared spectroscopy (FTIR). Cellulose 25:37-48

Ludvíková M, Griga M (2015) Transgenic flax/linseed (Linum usitatissimum L.)—expectations and reality. Czech J Genet Plant Breed 51(4):123-141

Lupoi JS, Smith-Moritz A, Singh S, McQualter R, Scheller HV, Simmons BA, Henry RJ (2015) Localization of polyhydroxybutyrate in sugarcane using Fourier-transform infrared microspectroscopy and multivariate imaging. Biotechnol Biofuels 8:98

Ma L, Zhang Z, Li J, Yang X, Fei B, Leung PHM, Tao X (2019) A new antimicrobial agent: poly (3-hydroxybutyric acid) oligomer. Macromol Biosci 19(5):e1800432

Murashige T, Skoog F (1962) A revised medium for rapid growth and bio assays with tobacco tissue cultures. Physiol Plant 15(3):473-497

Poirier Y, Nawrath C, Somerville C (1995) Production of polyhydroxyalkanoates, a family of biodegradable plastics and elastomers, in bacteria and plants. Biotechnology 13:142-150 
Popielarska-Konieczna M, Kozieradzka-Kiszkurno M, Swierczyńska J, Góralski G, Slesak H, Bohdanowicz J (2008) Ultrastructure and histochemical analysis of extracellular matrixsurface network in kiwifruit endosperm-derived callus culture. Plant Cell Rep 27(7):1137-1145

Popielarska-Konieczna M, Bohdanowicz J, Starnawska E (2010) Extracellular matrix of plant callus tissue visualized by ESEM and SEM. Protoplasma 247:121-125

Raza ZA, Abid S, Banat IM (2018) Polyhydroxyalkanoates: characteristics, production, recent developments and applications. Int Biodeterior Biodegrad 128:45-56

Rutkowska-Krause I, Mankowska G, Łukaszewicz M et al (2003) Regeneration of flax (Linum usitatissimum L.) plants from anther culture and somatic tissue with increased resistance to Fusarium oxysporum. Plant Cell Rep 22:110-116

Wróbel M, Zebrowski J, Szopa J (2004) Polyhydroxybutyrate synthesis in transgenic flax. J Biotechnol 107(1):41-54

Wróbel-Kwiatkowska M, Zebrowski J, Starzycki M, Oszmiański J, Szopa J (2007a) Engineering of PHB synthesis causes improved elastic properties of flax fibers. Biotechnol Prog 23(1):269-277

Wróbel-Kwiatkowska M, Starzycki J, Zebrowski J, Oszmiański J, Szopa J (2007b) Lignin deficiency in transgenic flax resulted in plants with improved mechanical properties. J Biotechnol 128:919-934

Wróbel-Kwiatkowska M, Skórkowska-Telichowska K, Dymińska L, Mączka M, Hanuza J, Szopa J (2009a) Biochemical, mechanical, and spectroscopic analyses of genetically engineered flax fibers producing bioplastic (poly- $\beta$-hydroxybutyrate). Biotechnol Prog 25:1489-1498
Wróbel-Kwiatkowska M, Żuk M, Szopa J, Dymińska L, Mączka M, Hanuza J (2009b) Poly-3-hydroxy butyric acid interaction with the transgenic flax fibers: FT-IR and Raman spectra of the composite extracted from a GM flax. Spectrochim Acta A 73:286-294

Wróbel-Kwiatkowska M, Czemplik M, Kulma A, Żuk M, Kaczmar J, Dymińska L, Hanuza J, Ptak M, Szopa J (2012) New biocomposites based on bioplastic flax fibers and biodegradable polymers. Biotechnol Prog 28:1336-1346

Wróbel-Kwiatkowska M, Kropiwnicki M, Żebrowski J et al (2019) Effect of mcl-PHA synthesis in flax on plant mechanical properties and cell wall composition. Transgenic Res 28:77-90

Yildiz M, Ozcan S, Er C (2002) The effect of different explant sources on adventitious shoot regeneration in flax (Linum usitatissimum L.). Turk J Biol 26:37-40

Yildiz M, Saglik Ç, Telci C, Erkilic EG (2011) The effect of in vitro competition on shoot regeneration from hypocotyl explants of Linum usitatissimum. Turk J Bot 35:211-218

Yildiz M (2012) The prerequisite of the success in plant tissue culture: high frequency shoot regeneration. Recent advances in plant in vitro culture. In Tech, Rijeka, pp 63-90

Zahir A, Nadeem M, Ahmad W et al (2019) Chemogenic silver nanoparticles enhance lignans and neolignans in cell suspension cultures of Linum usitatissimum L. Plant Cell Tissue Organ Cult 136:589-596

Publisher's Note Springer Nature remains neutral with regard to jurisdictional claims in published maps and institutional affiliations. 